\title{
Characterizing grooming behavior patterns and the influence of brush access on the behavior of group-housed dairy calves
}

\author{
K. C. Horvath and E. K. Miller-Cushon* \\ Department of Animal Sciences, University of Florida, Gainesville 32611
}

\section{ABSTRACT}

Group housing allows for dairy calves to perform social grooming behavior; however, the use of mechanical brushes may influence how calves groom themselves and others. Our objectives were, first, to characterize the bout characteristics of brush use, self-grooming, and allogrooming in calves and, second, to evaluate the effects of access to a rotating brush on grooming behavior. Holstein heifer and bull calves $(\mathrm{n}=32)$ were group-housed (4 calves/group) at 2 wk of life $(17 \pm 3 \mathrm{~d}$ of age; mean \pm standard deviation) and followed to wk 7 of life. Pens were assigned to receive either a rotating brush (BR; $\mathrm{n}=4$ pens) or no brush (CON; $\mathrm{n}=4$ pens). Behavior was recorded continuously for $12 \mathrm{~h}$ for 2 focal calves/pen on $2 \mathrm{~d}$ during wk 4, 6, and 7 of life. We performed a bout analysis by fitting a mixture of normal distributions to the $\log _{10}$-transformed frequency distribution of the intervals between recorded periods of behavior. We calculated bout criteria for brush use (125.9 s), allogrooming (125.9 s), and self-grooming (a mixture of 3 normal distributions provided the best fit for these data, providing 2 bout criteria: a shorter (micro) bout criterion of $50.1 \mathrm{~s}$ and a longer (macro) criterion of 1,000 s). Brush use was consistent across weeks, and calves used the brush for $20.5 \pm 6.1 \mathrm{~min} / 12$ $\mathrm{h}$ observation period, in $31.1 \pm 1.7$ bouts (mean $\pm \mathrm{SE}$ ). The frequency and duration of allogrooming bouts did not differ between treatments and across time. The $\mathrm{BR}$ calves tended to self-groom more than $\mathrm{CON}$ calves (16.3 vs. $14.3 \mathrm{~min} / 12 \mathrm{~h}$; $\mathrm{BR}$ vs. $\mathrm{CON} ; \mathrm{SE}=0.68$ ), and time spent self-grooming decreased across weeks. The frequency and duration of self-grooming micro bouts did not differ between treatments, but BR calves had shorter, more frequent self-grooming macro bouts (10.58 vs. 9.46 bouts; BR vs. $\mathrm{CON}$; $\mathrm{SE}=0.24)$. In summary, we determined that bout criteria could be fitted to grooming behaviors, which may be useful when characterizing these behaviors in future work, and that

Received July 27, 2018.

Accepted December 14, 2018.

*Corresponding author: emillerc@ufl.edu providing access to a rotating brush influenced selfgrooming behavior in group-housed calves.

Key words: dairy calf, grooming, allogrooming, bout criteria

\section{INTRODUCTION}

Self-grooming and allogrooming are frequently observed in cattle (Reinhardt et al., 1978) and provide a range of benefits including hygienic maintenance of a calf's body (Rich, 1973; Kohari et al., 2009), maintaining social relationships between individual cattle in a group (Reinhardt and Reinhardt, 1981; Færevik et al., 2007), and reducing the heart rate of the receivers (Sato and Tarumizu, 1993; Laister et al., 2011). Time spent performing self-grooming is influenced by aspects of the environment, such as the type of bedding provided (Panivivat et al., 2004), and internal factors, such as disease (Borderas et al., 2008), suggesting that self-grooming behavior is flexible and may be a good indicator of calf welfare. In semi-wild herds, social grooming occurs primarily between a cow and a calf (Reinhardt et al., 1986), but social grooming may also play a role in the formation of social relationships in weaned calves, as calves will spend more time grooming a familiar companion compared with an unfamiliar companion (Færevik et al., 2007).

Mechanical brushes provide an outlet for cows to satisfy natural grooming behavior and scratch hard-toreach areas of their body (DeVries et al., 2007), and young calves can also benefit from access to a brush. Weaned heifers will primarily use a brush to groom their head and neck area, but use on their trunk and posterior area varies by animal (Toaff-Rosenstein et al., 2017). Brushes are recommended for inclusion in housing due to their quick adoption and sustained use by adult cows (Georg and Totschek, 2001; DeVries et al., 2007). Increased opportunities for grooming behaviors as provided by mechanical brushes may be predicted to influence aspects of self-grooming or allogrooming, yet studies often describe brush usage and grooming in isolation. Thus, this study aimed to characterize patterns of grooming behavior in group-housed calves, and 
evaluate how access to a mechanical brush influences the development of these grooming behaviors over the preweaning and weaning period. We hypothesized that brush access would enhance grooming behavior, both self-directed and socially directed, and promote coat cleanliness, and that the performance of these behaviors may develop over time and respond to weaning.

Despite the importance of grooming behaviors, and discussion of them across the literature, the approach to quantifying these behaviors is inconsistent. Previous work has typically described grooming behaviors in terms of daily total activity time or percentages of time performed during an observation period, which may not capture all behavioral characteristics. Many behaviors can be described in terms of bouts, where the performance of the behavior is clumped in time, with brief pauses between the performance of a behavior within a single bout and longer time periods between bouts. However, determining the criteria for defining which time intervals fall within bouts and which fall between bouts requires consideration. Some previous authors have used various bout criteria for characterizing allogrooming, based on the ability to make reliable measurements between observers (Laister et al., 2011), in reference to previous works (Tresoldi et al., 2015; Šárová et al., 2016), or without clear justification (Sato et al., 1991; Krohn, 1994; Val-Laillet et al., 2009). However, more rigorous approaches to determining bout criteria have been developed, for example, to determine meal criteria for feeding behavior (Tolkamp et al., 1998; Yeates et al., 2001). Therefore, a secondary objective of this study was to evaluate patterns of brush use, allogrooming, and self-grooming, and determine the potential to more rigorously calculate bout criteria for the characterization of these behaviors.

\section{MATERIALS AND METHODS}

\section{Experimental Design}

Thirty-two Holstein heifer and bull calves were enrolled at the University of Florida Dairy Unit (Hague, FL), with study procedures approved by the University of Florida Animal Care and Use Committee. Calves were managed according to the standard operating procedures for this facility. They received $4 \mathrm{~L}$ of qualitycontrolled colostrum and were uniquely identified with radio-frequency identification ear tags at birth. At $2 \mathrm{wk}$ of life (17 $\pm 3 \mathrm{~d}$ of age; mean $\pm \mathrm{SD}$ ), consecutively born calves were moved to group pens (4 calves/group; 9.5 $\mathrm{m}^{2} /$ calf) deep-bedded with sand and located under an open-sided barn. Manure was removed daily from the pen. Pens were randomly assigned to receive either a rotating brush (DeLaval mini swinging brush; DeLaval, Tumba, Sweden; BR; $\mathrm{n}=4$ pens) or a control pen with no brush (CON; $\mathrm{n}=4$ pens).

All calves received $8 \mathrm{~L} / \mathrm{d}$ of milk replacer (SMI 28/15 BOVA/DFB Calf Milk Replacer, Southeast Milk, Inc., Mayo, FL) fed through an automated milk feeder (DeLaval CF1000X, DeLaval). Calves had ad libitum access to water and calf starter (Ampli-Calf Starter 20 Warm Weather, Purina Animal Nutrition LLC, Shoreview, MN) via a feeding trough. Beginning at $47 \pm 3$ d of age, calves were weaned through a 10-d step-down program $(8$ to $2 \mathrm{~L} / \mathrm{d})$, and calves were fully weaned at $57 \pm 3 \mathrm{~d}$ of age. A veterinarian monitored the health of the calves weekly, and calves were healthy for the duration of the experiment.

\section{Data Collection}

To observe behavior, video cameras (high definition day/night cameras; AXIS M2026-LE camera, Axis Communications Inc., Lund, Sweden) were mounted above each pen. Behavioral data were collected for 2 consecutive days at each of 2 time points during the preweaning stage, to assess how behavior develops during this period (wk 4; 28-29 $\pm 4 \mathrm{~d}$ of age, and wk 6; 43-44 $\pm 2 \mathrm{~d}$ of age), and for 2 consecutive days at one time point during weaning (wk 7; 50-51 \pm 3 d of age). For each pen, the behaviors of 2 focal animals (heifer calves; identified on video by unique coat markings) were recorded continuously within daylight hours (for $12 \mathrm{~h} ; 0600$ to $1800 \mathrm{~h}$ ). We recorded behavior start and end times using Behavioral Observation Research Interactive Software (BORIS; Friard and Gamba, 2016), which logged times with $1 / 100 \mathrm{~s}$ accuracy. Behaviors observed were brush use (defined as any body part in contact with the brush), self-grooming (licking or mouth movement in contact with any part of the calf's own body, or scratching with foot), and allogrooming (licking or mouth movement directed toward any part of another calf's body or head). Self-grooming and allogrooming were considered to be mutually exclusive, but brush use could occur at the same time as either self-grooming or allogrooming. A total of 4 observers were used to characterize these behaviors from video (inter-observer reliability was calculated for one day of video collection, with Cohen's kappa calculated in BORIS exceeding 0.85 for all observer comparisons).

To assess effects of brush use on calf cleanliness, a coat cleanliness score was recorded weekly using a 4-point scale adapted from Panivivat et al. (2004). For analysis, data were collapsed into 2 categories of clean ( 0 ; no dirt on thighs or body) or dirty ( 1 ; dirt present on thighs or body). 


\section{Behavior Bout Analysis}

Bout analysis was performed separately for each behavior (brush use, allogrooming, and self-grooming) both per individual calf (with data pooled across the 3 observation periods; six 12-h periods of behavioral data) and for all data combined (with data pooled across all calves and observation periods). Weekly subsets of data (with data pooled across all calves, for two 12-h time periods per calf) were also screened for evidence of week effects; in the absence of clear differences by week we did not further evaluate these data, as modeling these smaller subsets of data becomes less rigorous and we were not able to statistically test for week effects in bout criteria (which would have required subdividing weekly subsets of data by calf, which yielded data sets too small to model). Data from all observed calves (n $=16$ calves; 2 focal calves/pen) were included for bout analysis for allogrooming and self-grooming, whereas bout analysis for brush use was limited to calves in pens provided a brush ( $\mathrm{n}=8$ calves).

Similar to approaches taken in characterizing feeding bouts in cattle (DeVries et al., 2003), we took the $\log _{10^{-}}$ frequency distribution of the interval lengths between durations of time that each individual was performing each behavior (brush use, self-grooming, and allogrooming), over each day of observation. Bout criteria were calculated by fitting a mixture of normal distributions to the distributions of $\log _{10}$-transformed intervals using the method of exact maximum likelihood (using the $\mathrm{R}$ package mixdist; Macdonald and $\mathrm{Du}, 2018)$. This method uses the mixed probability density function:

$$
\mathrm{g}(x \mid \pi, \mu, \sigma)=\pi_{1} f\left(x \mid \mu_{1}, \sigma_{1}\right)+\ldots+\pi_{k} f\left(x \mid \mu_{k}, \sigma_{k}\right),
$$

where $\mathrm{g}$ is a weighted sum of $k$ component densities, with proportions $\pi$, means $\mu$, and standard deviations $\sigma$. For brush use and allogrooming, a mixture of 2 normal distributions $(k=2)$ was fitted to the data. These 2 distributions were interpreted as those within bouts and those between bouts. Based on conditional probabilities generated within the mixdist package, the bout criterion was determined as the point at which the distribution curve corresponding to the shorter intervals intersected the distribution curve corresponding to the longer intervals. An example of this analysis is provided in Supplemental Files S1, S2, and S3 (https://doi.org/ 10.3168/jds.2018-15460).

For self-grooming, visual inspection suggested the presence of 3 distributions in the pooled data and in the majority of individual calf data, and therefore both a mixture of 2 and 3 normal distributions were fitted to the data. Based on chi-squared goodness of fit sta- tistics calculated within mixdist, 3 normal distributions provided the best fit for most individual calves (10 of the 12 calves whose data we were able to model) and for the pooled data. To further evaluate the biological significance of this analysis, bout characteristics (as described below) were calculated using both the bout criterion corresponding to the intersection between the first and second distribution curve (a shorter micro bout criterion), as well as the criterion corresponding to the intersection between the second and third normal distribution (a longer macro bout criterion).

Based on our determined bout criteria, bout characteristics were calculated for each 12 -h observation period as bout frequency (counted as the number of intervals between periods of observed behavior that exceeded the criterion), bout time (sum of duration performing the behavior and intervals shorter than the bout criterion), and bout duration (bout time divided by bout frequency).

\section{Statistical Analysis}

It was not possible to fit the interval distribution for several individual calves (due to insufficient data points across the $6 \mathrm{~d}$ of data collection, for those calves that performed behaviors less frequently); individual bout criteria were therefore determined for a total of 6 (of 8 total) calves for brush use, 9 (of 16 total; 5 BR and 4 $\mathrm{CON}$ ) for allogrooming, and 12 (of 16 total; $7 \mathrm{BR}$ and $5 \mathrm{CON}$ ) for self-grooming. The specific calves whose data we could not model varied between behaviors. To assess how calculated bout frequency was influenced by different estimates of the bout criterion (i.e., those calculated by calf or from pooled data), we regressed within-calf measures based on calf-level criteria on those based on the pooled criterion (using PROC REG in SAS; version 9.4, SAS Institute Inc., Cary, NC). The intercept was tested for a difference from 0 , and the slope was tested for a difference from 1. Further, to assess possible effects of brush access on self-grooming and allogrooming bout structure, we tested for treatment differences in bout criteria (using a $t$-test; PROC TTEST in SAS) using the individual calf data that we were able to model.

Bout characteristics were summarized by pen and observation period (wk 4, 6, and 7). These data were screened for normality (using PROC UNIVARIATE in SAS) and compared between treatments using the MIXED procedure of SAS in a general linear mixed model with week as a repeated measure. The model included the fixed effect of treatment, week, and treatment by week interaction and the random effect of pen. The variance-covariance matrix structure was selected 
for each behavioral measure based on best fit according to the Schwarz's Bayesian information criterion (either the autoregressive structure or compound symmetry provided the best fit for all behavioral measures). Due to the binomial nature of the data, calf cleanliness was analyzed by week using the 2-sided Fisher's exact test (using PROC FREQ in SAS).

All values reported are least squares means. Significance was declared at $P<0.05$, and trends were reported if $0.05<P \leq 0.10$.

\section{RESULTS}

\section{Determining Grooming Bout Criteria}

The individual bout criteria ranged among calves from 63.1 to $199.5 \mathrm{~s}$ for brush use and from 50.1 to $316.2 \mathrm{~s}$ for allogrooming. Pooled bout criteria were $125.9 \mathrm{~s}$ for brush use (Figure 1a) and $125.9 \mathrm{~s}$ for allogrooming (Figure 1b). For self-grooming, the individual bout micro criteria, based on the intersection between the first 2 distribution curves, ranged from 31.6 to 63.1 s. For the 10 (of 12 calves modeled) whose data were fitted using a mixture of 3 normal distributions, the longer macro bout criteria, based on the intersection between the second 2 distribution curves, ranged from 631.0 to $1,584.9 \mathrm{~s}$. The pooled criteria for self-grooming were $50.1 \mathrm{~s}$ (for the micro bout) and $1,000 \mathrm{~s}$ (for the macro bout; Figure 1c).

We found no effect of treatment on self-grooming bout criteria (micro: 43.1 vs. 48.6 s; $\mathrm{BR}$ vs. $\mathrm{CON}$; $\mathrm{SE}=$ $4.30 ; \mathrm{t}=-1.14$, df $=10 ; P=0.28$; or macro: 988.6 vs. $1,179.4 \mathrm{~s} ; \mathrm{BR}$ vs. $\mathrm{CON} ; \mathrm{SE}=133.7 ; \mathrm{t}=-1.04, \mathrm{df}=8$; $P=0.33)$ or allogrooming bout criteria (120.3 vs. 134.8 $\mathrm{s} ; \mathrm{BR}$ vs. $\mathrm{CON} ; \mathrm{SE}=45.0 ; \mathrm{t}=-0.3, \mathrm{df}=7 ; P=0.77)$.

The relationships between bout frequency estimates based on pooled and calf-level bout criteria (shown for self-grooming micro bouts) are presented for each behavior in Figure 2. In every case, the intercept did not differ from 0 , and the slope did not differ from 1 $(P>0.1)$. Because we found no effect of treatment on bout criteria, and the use of the pooled bout criterion versus the criteria calculated per calf yielded similar estimates of bout frequency for all behaviors, we used the pooled bout criterion (Figure 2) to calculate bout characteristics for all behaviors. An example of continuously recorded behavior and defined bouts, based on pooled criteria, with intra-bout intervals clearly visible is shown for each behavior in Figure 3, and in Figure 4 we provide an example of a calf engaging in all 3 behaviors interspersed during a 1-h time period, in which bouts of brush use, allogrooming, and micro and macro bouts of self-grooming are evident, and visually represented with solid lines.

\section{Effect of Access to a Rotating Brush on Grooming Behavior}

Calves that had access to a brush used it for about 20 min per 12 -h observation period (Table 1 ; minimum
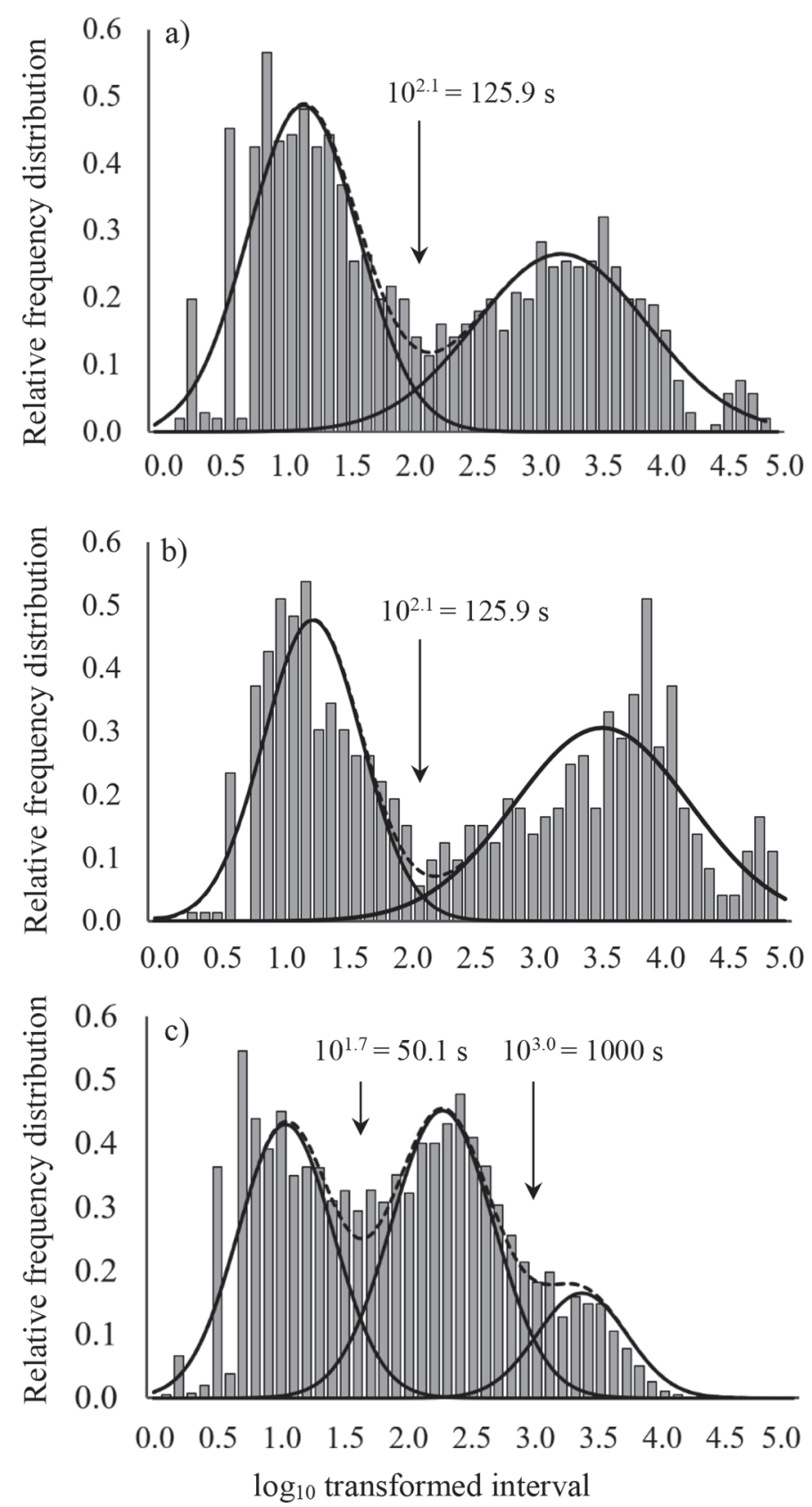

Figure 1. $\log _{10}$-transformed relative frequency distribution of the intervals between the periods of time spent (a) using the rotating brush, (b) allogrooming, and (c) self-grooming, fitted with a mixture of normal distributions. Data presented are summarized for 16 calves ( 8 calves for brush data) over $6 \mathrm{~d}$ of observation across 3 time periods: 2 d starting at d 28-29 \pm 4 d of life, $43-44 \pm 2$ d of life, and 50-51 $\pm 3 \mathrm{~d}$ of life. The solid lines represent the contribution of individual distributions to the overall probability density (dashed line). 
$1.0 \mathrm{~min} / 12 \mathrm{~h}$ and maximum $65.5 \mathrm{~min} / 12 \mathrm{~h}$ ). Although total time spent using the brush did not differ significantly across observation weeks, brush bout frequency changed over time (increasing from wk 4 to 6 and then decreasing during weaning in wk $7 ; 7.63$ vs. 13.69 vs.
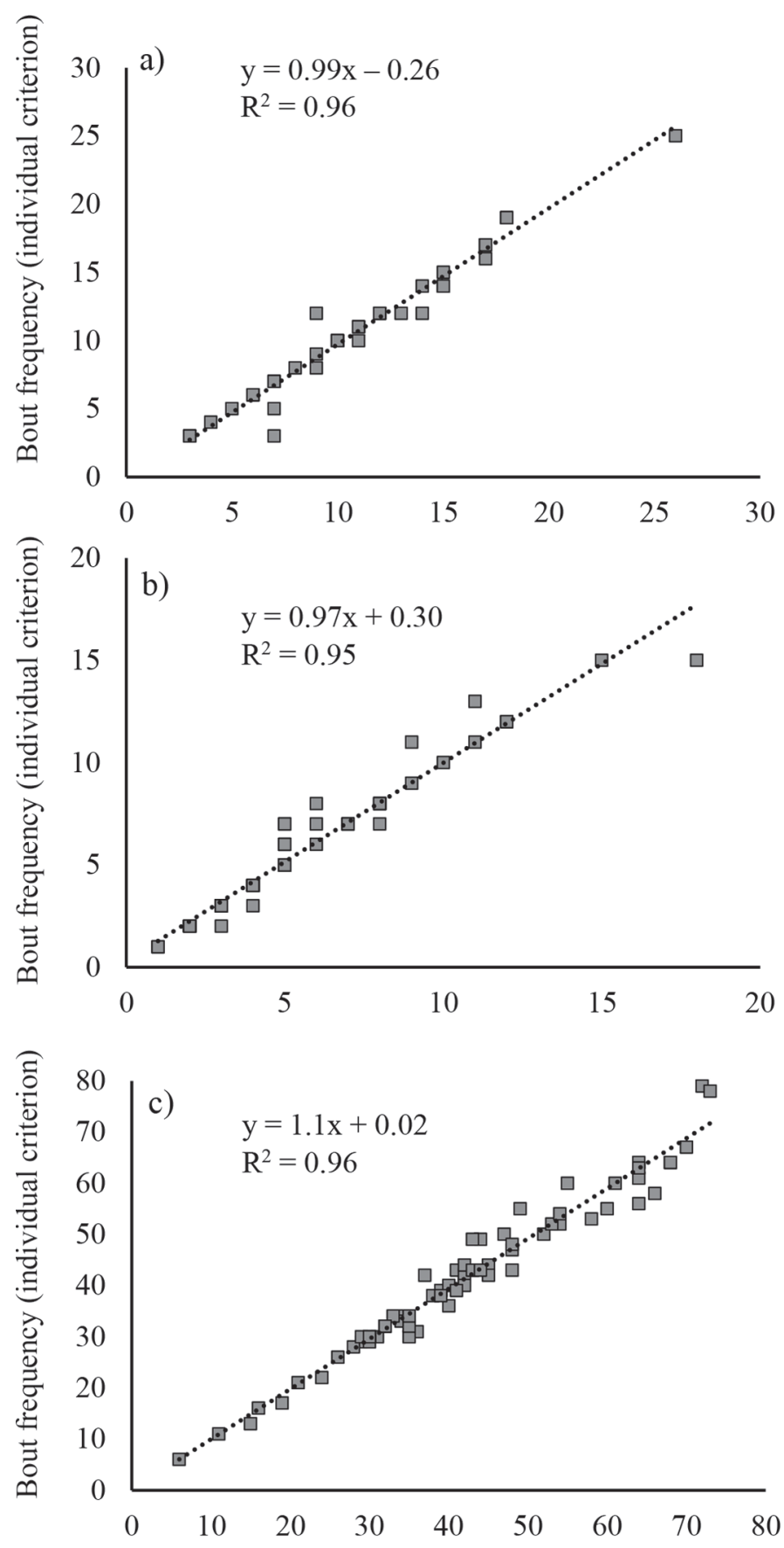

Bout frequency (pooled criterion)

Figure 2. Association between bout frequency calculated using a pooled bout criterion and with individual calf bout criteria for (a) brush usage, (b) allogrooming, and (c) self-grooming (micro bouts).
9.75 bouts; wk 4 vs. wk 6 vs. wk 7) while brush bout duration remained consistent (Table 1).

Calves provided a brush tended to spend more time overall self-grooming (Table 1; minimum $0.75 \mathrm{~min} / 12$ $\mathrm{h}$ and maximum $40.0 \mathrm{~min} / 12 \mathrm{~h}$ ). Characteristics of micro bouts, based on the shorter bout criterion (shown in Figure 1c), did not differ between treatments, but the percentage of time spent grooming during micro bouts tended to be greater for calves with a brush. Time spent using the brush decreased between weeks, with micro bouts becoming shorter and tending to decrease in frequency. Calves provided a brush had more frequent, shorter macro bouts, as defined based on the longer bout criterion, and macro bout characteristics did not differ over time. Providing a brush had no effect on the duration or frequency of allogrooming, and allogrooming behavior was similar across time (Table 1). Calves provided a brush were cleaner during weaning in wk 7 (0.5 vs. 0.94 cleanliness score; $\mathrm{SE}=0.09 ; P=$ $0.02)$, but cleanliness did not differ between treatments during the preweaning observation wk 3 to $6(P>0.6)$.

\section{DISCUSSION}

Grooming is a natural behavior exhibited by cattle, which appears to have some important roles including hygienic maintenance of the coat (Rich, 1973; Kohari et al., 2009) and as a means to maintain social relationships (Færevik et al., 2007). Provision of a rotating brush may allow for increased grooming behaviors and has been explored in some recent work in cattle of different ages. However, reports of brush use across the literature are variable and could depend on the age of the calf, stocking density, and competition for access to the brush, and the type of brush provided. Whereas we found that brush use was stable across time, others have reported changing usage over time. For example, Pempek et al. (2017) reported that dairy calves increased use of a stationary brush (from 2.5 to 9 min, per 12-h observation period, across the first 63 $\mathrm{d}$ of life). A range in brush use times has also been reported in calves offered a mechanical rotating brush in previous studies. In calves under 2 wk of age, Zobel et al. (2017) reported that the duration of brush use was 27 min per 20 -h observation period, which is consistent with the present study, whereas shorter durations of brush use have been reported in older calves (e.g., 9.1 $\mathrm{min} / \mathrm{d}$ in calves aged 40-71 d; Georg et al., 2007). In general, the considerable amount of time that calves spend using a rotating brush suggests that it may be beneficial for calf welfare. Brush use has been suggested to potentially be a redirected behavior associated with maternal grooming and care in both cows (Mandel and Nicol, 2017) and calves (Zobel et al., 2017). Reports 

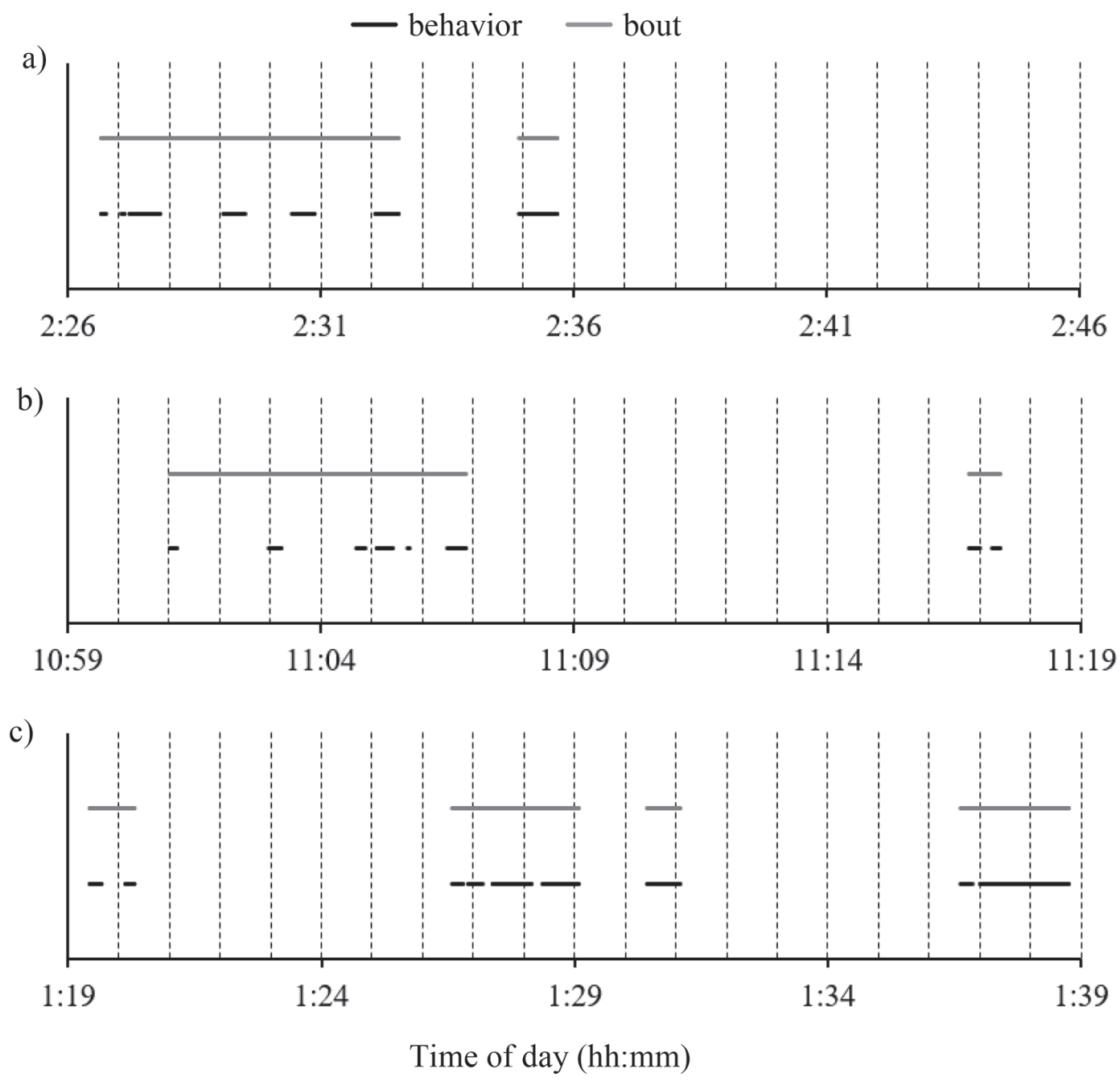

Figure 3. Examples of continuously recorded behavior and corresponding bout definitions from different calves over a 20-min period for (a) using the rotating brush (bout criterion of $125.9 \mathrm{~s}$ ), (b) allogrooming (bout criterion of $125.9 \mathrm{~s}$ ), and (c) self-grooming (micro bout criterion of $50.1 \mathrm{~s})$. Vertical dashes represent 1-min marks.

of maternal grooming time between the cow and the calf vary across the literature; for example, Kohari et al. (2009) reported 2.7 to $25.1 \mathrm{~min} / 8 \mathrm{~h}$ observation period of grooming between Japanese beef cows and their calves and Braghieri et al. (2011) reported $7.2 \mathrm{~min} / 7 \mathrm{~h}$ observation period of grooming between Mediterranean beef cows and their calves. Additionally, dairy cows that experienced a daily 12 -h separation from their calf spent approximately 12 min grooming their calf in the first $2 \mathrm{~h}$ after reuniting with their calf (Johnsen et al., 2015). These results suggest that the amount of time a calf would receive maternal grooming may be similar to time spent using a rotating brush. The possibility that brush use may act as a partial substitute for maternal care should be investigated further.

Characteristics of brush use beyond duration of use are not reported consistently across the literature. Zobel et al. (2017) reported a bout duration of $17.8 \mathrm{~s} /$ bout and frequency of 94 bouts per $20 \mathrm{~h}$, where bout appeared to be equated with contact frequency. In contrast, adult cows appear to visit the brush less frequently (4.5 visits per d; Mandel et al., 2013 and 7.7 visits per d; DeVries et al., 2007). Comparisons across studies describing brush use are difficult because varying approaches to describing this behavior have been taken. For example, Mandel et al. (2013) recorded whether a cow used the brush during each 5-min interval across a 24 -h period, which may not accurately capture brush visit characteristics. Zobel et al. (2017) recorded behavior continuously and reported a high number of visits to the brush (roughly $3 \times$ the frequency of brush bouts that we reported, based on our bout criteria). No previous work to our knowledge has approached characterizing brush use based on a rigorously defined bout criteria, which 
Table 1. Description of self-grooming and allogrooming behavior and bout characteristics of calves on different brush treatments: provided a rotating brush (brush) or no brush (control), recorded continuously over a 12-h observation period (0600 to $1800 \mathrm{~h}$ ) on 2 consecutive days in wk 4,6 , and 7 of life

\begin{tabular}{|c|c|c|c|c|c|c|}
\hline \multirow[b]{2}{*}{ Behavior } & \multicolumn{2}{|c|}{ Treatment } & \multirow[b]{2}{*}{$\mathrm{SE}$} & \multicolumn{3}{|c|}{$P$-value ${ }^{1}$} \\
\hline & Brush & Control & & $\mathrm{T}$ & W & $\mathrm{T} \times \mathrm{W}$ \\
\hline \multicolumn{7}{|l|}{ Brush use } \\
\hline Duration (min) & 20.5 & - & 6.2 & - & 0.29 & - \\
\hline Bout frequency (no.) & 10.4 & - & 1.6 & - & 0.02 & - \\
\hline Bout duration (s/bout) & 154.2 & - & 19.8 & - & 0.73 & - \\
\hline \multicolumn{7}{|l|}{ Self grooming ${ }^{2}$} \\
\hline Duration (min) & 16.3 & 14.3 & 0.68 & 0.08 & 0.007 & 0.73 \\
\hline Micro bout frequency (no.) & 41.0 & 41.0 & 1.94 & 0.99 & 0.06 & 0.60 \\
\hline Micro bout duration (s/bout) & 35.3 & 33.9 & 1.88 & 0.61 & 0.006 & 0.82 \\
\hline Grooming during micro bouts (\%) & 68.6 & 64.9 & 0.013 & 0.09 & 0.005 & 0.65 \\
\hline Macro bout frequency (no.) & 10.6 & 9.5 & 0.24 & 0.02 & 0.91 & 0.31 \\
\hline Macro bout duration (min/bout) & 15.1 & 18.9 & 1.0 & 0.04 & 0.12 & 0.46 \\
\hline Grooming during macro bouts (\%) & 10.5 & 8.5 & 0.56 & 0.04 & 0.027 & 0.70 \\
\hline \multicolumn{7}{|l|}{ Allogrooming } \\
\hline Duration (min) & 3.8 & 3.0 & 0.88 & 0.53 & 0.26 & 0.82 \\
\hline Bout frequency (no.) & 4.98 & 4.02 & 0.84 & 0.45 & 0.46 & 0.86 \\
\hline Bout duration (s/bout) & 67.7 & 57.1 & 8.31 & 0.40 & 0.94 & 0.84 \\
\hline
\end{tabular}

${ }^{1} P$-values refer to the fixed effects of treatment $(\mathrm{T})$, week $(\mathrm{W})$, and treatment by week interaction $(\mathrm{T} \times \mathrm{W})$.

${ }^{2}$ We defined micro bouts according to the shorter criterion of $50.1 \mathrm{~s}$, and macro bouts according to the longer criterion of $1,000 \mathrm{~s}$.

allows a clear definition of individual bout frequency and duration. As visually depicted in Figure 3a, use of the bout criterion we calculated provided a biologically meaningful distinction between brief pauses during periods of brush use that comprise a single bout, and longer pauses between bouts.

Allogrooming has been described as a means to form and maintain relationships in a herd (Reinhardt and
Reinhardt, 1981; Færevik et al., 2007), but the development of this behavior in preweaned calves has not been well studied. In lactating cows, Val-Laillet et al. (2009) reported allogrooming occurring for $320 \mathrm{~s} / \mathrm{d}$, in 5.7 bouts lasting 35 to $88 \mathrm{~s} /$ bout. Sato et al. (1991) observed 12- to 15-mo-old Holstein heifers and steers for $2 \mathrm{~h}$ before sunset and reported allogrooming durations of $210 \mathrm{~s} / \mathrm{h}$, and a frequency of 4.8 bouts lasting 43

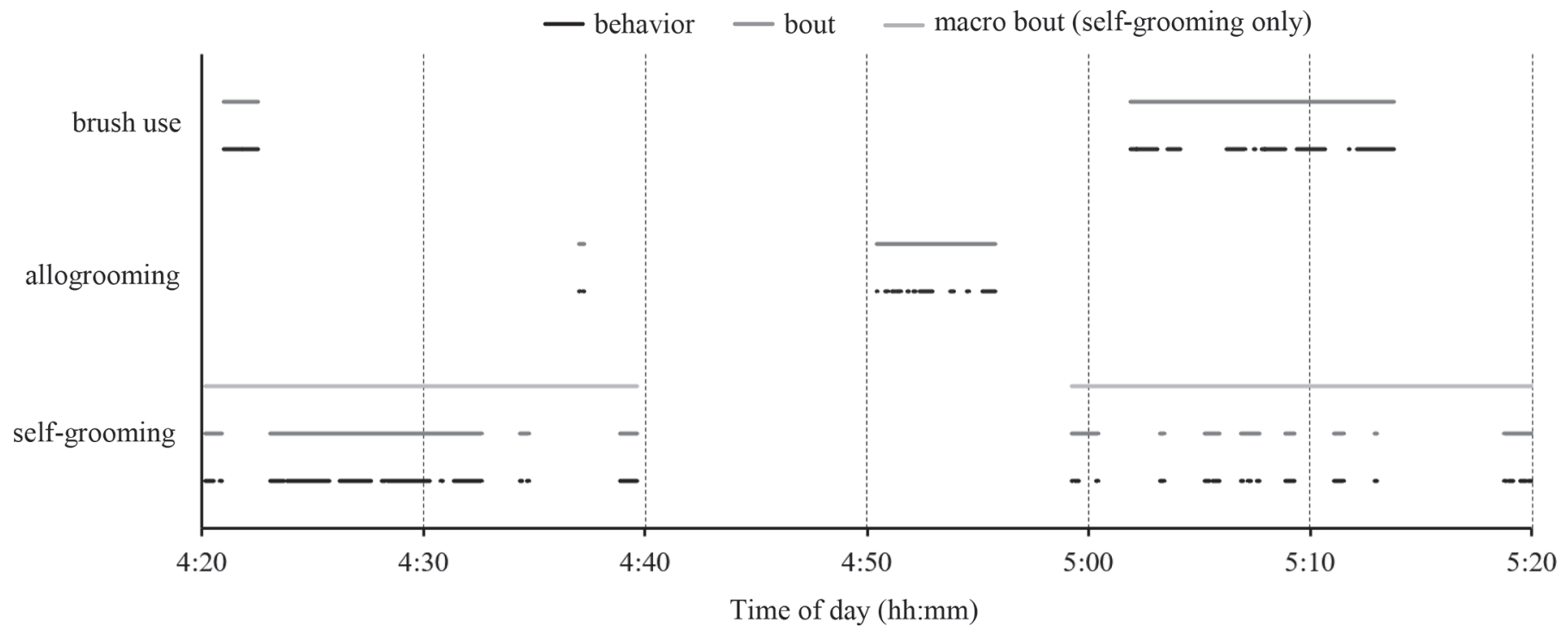

Figure 4. Example of continuously recorded behavior and corresponding bout definitions from a single calf over a 1-h time period where all 3 focal behaviors (brush use, allogrooming, and self-grooming) were performed. For self-grooming, a third line (light gray) represents the macro bouts defined by the criterion corresponding to the intersection between the second and third normal distributions fitted to the data (see Figure 2c). Vertical dashes represent 10-min marks. 
$\mathrm{s} /$ bout. In previous work, we found that calves spent approximately $2 \mathrm{~min}$ allogrooming in the first $7 \mathrm{~h}$ after introduction to a social group after weaning (Horvath and Miller-Cushon, 2018). In general, we found that calves in the present study spent less time allogrooming compared with previous reports of allogrooming in older animals, suggesting that this behavior may develop more as calves mature or may depend on time spent in social housing and familiarity of social companions. We predicted that provision of the brush may enhance allogrooming, yet saw no differences between treatments, at least during our period of daytime observation, possibly due to the limited performance of this behavior. We encourage further research to explore the development of allogrooming in calves over time and in response to different housing factors, with a more rigorous approach of identifying all individuals involved as a recipient and actor in grooming interactions.

Different approaches to defining allogrooming bouts have been taken across the literature. An allogrooming bout criteria of $30 \mathrm{~s}$ has been used in lactating cows (Val-Laillet et al., 2009), year-old heifers and bulls (Sato et al., 1991), and pregnant 2-yr-old heifers (Tresoldi et al., 2015). Other studies have used a bout criterion of 10 s in beef cattle (Šárová et al., 2016) and lactating cows (Laister et al., 2011). Krohn (1994) used a bout criterion of $5 \mathrm{~s}$ in dairy cattle. Utilizing a cut-off value during observation may be useful, such that a new bout can be defined when the actor has stopped grooming the reactor for at least that period of time. However, it is not clear whether these previously used bout criteria accurately reflect the characteristics of the behavior. Laister et al. (2011) describe selection of their $10 \mathrm{~s}$ bout criterion based on the ability to achieve high interobserver reliability, whereas others have not provided a clear justification for selecting different criteria. Based on our bout analysis, a bout criterion closer to $2 \mathrm{~min}$ in duration may more accurately reflect the allogrooming behavior of young calves. We encourage further work to more rigorously analyze bout characteristics to determine a valid bout criterion for allogrooming in adult cows and older heifers.

Self-grooming is noted frequently in calves, and is important in maintaining the hygiene of the animal (Rich, 1973; Kohari et al., 2009). This behavior also responds to various physiological and environmental factors, suggesting a possible link with calf comfort and welfare. For example, a reduction in duration of self-grooming has been reported following experimental disease challenge models that induce sickness behavior (Borderas et al., 2008; Hixson et al., 2018). Additionally, bedding material affects time spent self-grooming, with calves performing more self-grooming when housed on sand and rice hulls compared with long wheat straw, possibly due to the bedding material sticking to their coat (Panivivat et al., 2004). Reported durations of time spent self-grooming vary widely across the literature. For example, we previously found that individually housed calves spent more time self-grooming than calves in the present study (over $20 \mathrm{~min} / 8 \mathrm{~h}$ observation period; Horvath and Miller-Cushon, 2017), whereas recently weaned heifers spent very little time self-grooming during the first week of social grouping ( $5 \mathrm{~min} / 7 \mathrm{~h}$ observation period; Horvath and Miller-Cushon, 2018). These variable results suggest that a range of factors, possibly including complexity of environment and calf affective state, may influence self-grooming. In the present study, we found that provision of a brush tended to increase the amount of time calves spent self-grooming, suggesting that brush access may stimulate grooming behavior to some extent. Given other positive effects of brush provision, we think this could suggest that increased self-grooming behavior may, in some contexts, reflect improved welfare. We encourage further work exploring the development of self-grooming in dairy calves and links between self-grooming and other metrics of calf welfare, particularly over longer periods of time and throughout 24-h observation periods. For example, further work exploring the temporal relationship between bouts of self-grooming and other grooming activities may provide some insight into the function of grooming activities.

Our bout analysis of self-grooming suggested 2 different meaningful cutoff values for the determination of bouts. An example of how these 2 criteria grouped the continuously recorded behavior is shown in Figure 4. In general, self-grooming was comprised of many brief actions (e.g., scratching or licking with short pauses between), which were clumped using the micro bout criteria. However, grooming activity also appeared to be spread over longer periods of time, and was interspersed with other activities such as brush use and allogrooming (as shown in Figure 4) as well as, anecdotally, feeding or drinking. As such, the longer bouts defined using the macro bout criteria may reflect prolonged periods of increased self-grooming activity. We found that calves provided a brush had more frequent and shorter macro bouts of self-grooming, which were comprised of relatively more time spent grooming. This suggests that opportunity for brush use, and possibly increased environmental complexity in general, may cause calves to allocate their time differently between activities and influence behavioral bout structure. Selfgrooming has been characterized in calves previously using time sampling methods (e.g., 10-min intervals; Chua et al., 2002, and 15-min intervals, Panivivat et al., 2004). Based on the duration of our described macro bouts, these time sampling methods may be 
likely to catch a long bout at least once; however, due to the presence of pauses during these bouts (Figure 4), an instantaneous scan sample may not coincide with performance of the behavior. Therefore, we suggest that time sampling methods to characterize grooming behavior be validated before use.

It is interesting to note the visual similarities between the frequency distributions of intervals between periods of brush use and allogrooming. Further, the bout criteria for both behaviors, as determined from the pooled data, were the same. Both of these behaviors had 2 well-defined distributions corresponding to short pauses within bouts and long pauses between bouts, with fewer pauses of intermediate length. In contrast, the frequency distribution of intervals between periods of self-grooming were less well defined, with less of a clear separation between the distributions (Figure 1c). In feeding behavior, the bout structure depends on the concept of satiety, where the odds of a new meal beginning increases with time since the last meal and divides the behavior into discrete bouts with relatively few short pauses between meals (Tolkamp et al., 1998). Allogrooming and brush use exhibited a bimodal distribution which resembled that seen in feeding behavior (e.g., Tolkamp et al., 1998; DeVries et al., 2003), although the frequency distributions were centered at shorter time intervals. Although brush use and grooming may not be subject to the same internal controls as feeding, we might expect that, in general, a behavior would be clustered in bouts, with relatively few short pauses between bouts, if there is any cost or barrier to initiating the behavior. For example, when a brush is located further from a feeding area, cows have been noted to decrease their use of the brush when experiencing heat stress (Mandel et al., 2013), metritis (Mandel et al., 2017), or lameness (Mandel et al., 2018), whereas brush use is maintained during these conditions of stress or illness if the brush is easier to access and located near the feeding area. Although we did not observe competition for access to the brush in the present study, increasing stocking density and competition for access to a brush may be similarly expected to influence brush bout characteristics. For allogrooming, the barrier to initiating the behavior may depend primarily on the presence of social companions, as social licking events have been found to increase when the stocking density increases in pregnant dairy heifers (Tresoldi et al., 2015).

Interestingly, we found that calves that had access to a brush were cleaner during the week of weaning. Across different bedding systems, Panivivat et al. (2004) described decreasing calf cleanliness over time, suggesting that maintaining cleanliness may be an increasing challenge in later weeks of the preweaning period. As such, positive effects of brush access on calf cleanliness may then be more likely to become apparent later in the preweaning period. DeVries et al. (2007) also reported anecdotally that cows with brushes appeared cleaner than those without. This suggests that brushes may have a positive effect on calf coat cleanliness, either directly from effects of the brush in removing dirt or indirectly through stimulating more self-grooming behavior, as we observed in the present study in calves provided a brush. We encourage future work to assess potential health consequences of this effect in larger groups of calves in different housing environments.

Overall, we were able to determine bout criteria to characterize bouts of brush use, allogrooming, and self-grooming, and similar approaches in future work may allow for more rigorous and informative description of these behaviors. Where possible, we encourage independent calculation of bout criteria specific to data collected for different data sets, as bout criteria and structure may vary over time and in response to varying management scenarios. We also found that provision of a brush influenced self-grooming during our daytime observation period, possibly stimulating increased expression of this behavior and shaping bout patterns. We encourage further work to evaluate how calf management and housing factors influence grooming behaviors, and the importance of expressing natural grooming behavior for calf welfare.

\section{ACKNOWLEDGMENTS}

We thank the staff at the University of Florida Dairy Unit, Catherine Hixson, and our undergraduate research students (Rachel Brick, Alexis Brocious, Alyssa Carillo, Tessa Fackler, Kaitlin Gingerich, Mackenzie Holmes, Megan McDowell, Giuliana Pezzella, Taylor Pollak, and Sam Stella) for their technical assistance. KH was supported by a Matching Assistantship through the Department of Animal Sciences, University of Florida. This research was supported by the USDA National Institute of Food and Agriculture, Hatch Multistate project 1012277.

\section{REFERENCES}

Borderas, T. F., A. M. De Passillé, and J. Rushen. 2008. Behavior of dairy calves after a low dose of bacterial endotoxin. J. Anim. Sci. 86:2920-2927.

Braghieri, A., C. Pacelli, A. Girolami, and F. Napolitano. 2011. Time budget, social and ingestive behaviours expressed by native beef cows in Mediterranean conditions. Livest. Sci. 141:47-52.

Chua, B., E. Coenen, D. J. Van, and D. M. Weary. 2002. Effects of pair versus individual housing on the behavior and performance of dairy calves. J. Dairy Sci. 85:360-364.

DeVries, T. J., M. Vankova, D. M. Veira, and M. A. G. von Keyserlingk. 2007. Short communication: Usage of mechanical brushes by lactating dairy cows. J. Dairy Sci. 90:2241-2245. 
DeVries, T. J., M. A. G. von Keyserlingk, D. M. Weary, and K. A. Beauchemin. 2003. Measuring the feeding behavior of lactating dairy cows in early to peak lactation. J. Dairy Sci. 86:3354-3361.

Færevik, G., I. L. Andersen, M. B. Jensen, and K. E. Bøe. 2007. Increased group size reduces conflicts and strengthens the preference for familiar group mates after regrouping of weaned dairy calves (Bos taurus). Appl. Anim. Behav. Sci. 108:215-228.

Friard, O., and M. Gamba. 2016. BORIS: A free, versatile open-source event-logging software for video/audio coding and live observations. Methods Ecol. Evol. 7:1325-1330.

Georg, H., D. Jahn-Falk, and G. Ude. 2007. Technology against boredom: Is an automatic grooming brush accepted by calves? Cattle Husb. 62:166-167.

Georg, H., and K. Totschek. 2001. Investigation of an automatic brush for dairy cows. Cattle Prod. 56:260-261.

Hixson, C. L., P. D. Krawczel, J. M. Caldwell, and E. K. Miller-Cushon. 2018. Behavioral changes in group-housed dairy calves infected with Mannheimia haemolytica. J. Dairy Sci. 101:10351-10360.

Horvath, K. C., and E. Miller-Cushon. 2018. Characterizing social behavior, activity, and associations between cognition and behavior upon social grouping of weaned dairy calves. J. Dairy Sci. 101:7287-7296.

Horvath, K. C., and E. K. Miller-Cushon. 2017. The effect of milkfeeding method and hay provision on the development of feeding behavior and non-nutritive oral behavior of dairy calves. J. Dairy Sci. 100:3949-3957.

Johnsen, J. F., A. M. de Passille, C. M. Mejdell, K. E. Bøe, A. M. Grøndahl, A. Beaver, J. Rushen, and D. M. Weary. 2015. The effect of nursing on the cow-calf bond. Appl. Anim. Behav. Sci. 163:50-57.

Kohari, D., S. Sato, and Y. Nakai. 2009. Does the maternal grooming of cattle clean bacteria from the coat of calves? Behav. Processes 80:202-204.

Krohn, C. C. 1994. Behaviour of dairy cows kept in extensive (loose housing/pasture) or intensive (tie stall) environments III. Grooming, exploration and abnormal behavior. Appl. Anim. Behav. Sci. $42: 73-86$

Laister, S., B. Stockinger, A. M. Regner, K. Zenger, U. Knierim, and C. Winckler. 2011. Social licking in dairy cattle-Effects on heart rate in performers and receivers. Appl. Anim. Behav. Sci. 130:8190.

Macdonald, P., and J. Du. 2018. mixdist: Finite Mixture Distribution Models. R package version 0.5-5. Accessed Jun. 11, 2018. https:// CRAN.R-project.org $/$ package $=$ mixdist

Mandel, R., H. Harazy, L. Gygax, C. J. Nicol, A. Ben-David, H. R. Whay, and E. Klement. 2018. Short communication: Detection of lameness in dairy cows using a grooming device. J. Dairy Sci. 101:1511-1517.

Mandel, R., and C. J. Nicol. 2017. Re-direction of maternal behaviour in dairy cows. Appl. Anim. Behav. Sci. 195:24-31.

Mandel, R., C. J. Nicol, H. R. Whay, and E. Klement. 2017. Short communication: Detection and monitoring of metritis in dairy cows using an automated grooming device. J. Dairy Sci. 100:5724-5728.
Mandel, R., H. R. Whay, C. J. Nicol, and E. Klement. 2013. The effect of food location, heat load, and intrusive medical procedures on brushing activity in dairy cows. J. Dairy Sci. 96:6506-6513.

Panivivat, R., E. B. Kegley, J. A. Pennington, D. W. Kellogg, and S. L. Krumpelman. 2004. Growth performance and health of dairy calves bedded with different types of materials. J. Dairy Sci. $87: 3736-3745$.

Pempek, J. A., M. L. Eastridge, and K. L. Proudfoot. 2017. The effect of a furnished individual hutch pre-weaning on calf behavior, response to novelty, and growth. J. Dairy Sci. 100:4807-4817.

Reinhardt, C., A. Reinhardt, and V. Reinhardt. 1986. Social behaviour and reproductive performance in semi-wild Scottish Highland cattle. Appl. Anim. Behav. Sci. 15:125-136.

Reinhardt, V., F. M. Mutiso, and A. Reinhardt. 1978. Social behaviour and social relationships between female and male prepubertal bovine calves (Bos indicus). Appl. Anim. Ethol. 4:43-54.

Reinhardt, V., and A. Reinhardt. 1981. Cohesive relationships in a cattle herd (Bos indicus). Behaviour 77:121-151.

Rich, G. B. 1973. Grooming and yarding of spring-born calves prevent paralysis caused by the Rocky Mountain wood tick. Can. J. Anim. Sci. 53:377-378.

Šárová, R., A. K. Gutmann, M. Špinka, I. Stěhulová, and C. Winckler. 2016. Important role of dominance in allogrooming behaviour in beef cattle. Appl. Anim. Behav. Sci. 181:41-48.

Sato, S., S. Sako, and A. Maeda. 1991. Social licking patterns in cattle (Bos taurus): Influence of environmental and social factors. Appl. Anim. Behav. Sci. 32:3-12.

Sato, S., and K. Tarumizu. 1993. Heart rates before, during and after allo-grooming in cattle (Bos taurus). J. Ethol. 11:149-150.

Toaff-Rosenstein, R. L., M. Velez, and C. B. Tucker. 2017. Technical note: Use of an automated grooming brush by heifers and potential for radiofrequency identification-based measurements of this behavior. J. Dairy Sci. 100:8430-8437.

Tolkamp, B. J., D. J. Allcroft, E. J. Austin, B. L. Nielsen, and I. Kyriazakis. 1998. Satiety splits feeding behavior into bouts. J. Theor. Biol. 194:235-250.

Tresoldi, G., D. M. Weary, L. C. P. M. Filho, and M. A. G. von Keyserlingk. 2015. Social licking in pregnant dairy heifers. Animals (Basel) 5:1169-1179.

Val-Laillet, D., V. Guesdon, M. A. G. von Keyserlingk, A. M. de Passillé, and J. Rushen. 2009. Allogrooming in cattle: Relationships between social preferences, feeding displacements and social dominance. Appl. Anim. Behav. Sci. 116:141-149.

Yeates, M. P., B. J. Tolkamp, D. J. Allcroft, and I. Kyriazakis. 2001. The use of mixed distribution models to determine bout criteria for analysis of animal behavior. J. Theor. Biol. 213:413-425.

Zobel, G., H. W. Neave, H. V. Henderson, and J. Webster. 2017. Use an automated brush and a hanging rope when pair-housed. Animals (Basel) 7:1-11. 